\title{
Comparison of Pre-Operative Endoscopic Retrograde Cholangiopancreatography Followed by Laparoscopic Cholecystectomy and Laparoscopic Cholecystectomy with Intraoperative Cholangiography in Acute Biliary Pancreatitis
}

\author{
Al-Ghouthani Jamal ${ }^{1} \&$ Alyounes Abdulmonem ${ }^{1}$ \\ ${ }^{1}$ Carol Davila University of Medicine and Pharmacy, Bucharest, Romania \\ Correspondence: Al-Ghouthani Jamal, Carol Davila University of Medicine and Pharmacy, Bucharest, Romania. \\ E-mail: jamalgothani@hotmail.com
}

Received: January 13, 2017 Accepted: February 22, 2017 Online Published: March 7, 2017

doi:10.5539/gjhs.v9n5p131 URL: https://doi.org/10.5539/gjhs.v9n5p131

\begin{abstract}
Aim: To compare pre-operative endoscopic retrograde cholangiopancreatography and laparoscopic cholecystectomy with laparoscopic cholecystectomy intra-operative cholangiogram and selective common bile duct exploration.

Methods: A cohort study involved 50 patients with the diagnosis of gall stone pancreatitis. The age ranged from 15 to 85 years (mean=50 yrs.), 15 patients were males and 35 were females. They were studied before and after the management. The study included patients who met criteria for gall stone pancreatitis, defined as upper abdominal pain with an elevated serum amylase, nausea or vomiting, and cholelithiasis by ultrasound examination. Fifty patients were selected and divided into two groups, those who underwent preoperative ERCP followed by laparoscopic cholecystectomy and those who had a laparoscopic cholecystectomy and intra-operative cholangiogram with surgical clearance of the common bile duct as needed.

Results: In our study, our patients involved in both groups, all of them had mild to moderate acute gallstone pancreatitis because all of them met 3 or less Ranson's criteria. In the preoperative ERCP and laparoscopic Cholecystectomy group, 8 patients $(32 \%)$ were found to have choledocholithiasis and underwent endoscopic sphincterotomy for stone extraction. Stones were extracted from 5 patients $20 \%$. The other 3 patients (12\%) ERCP failed to extract the stones. Another 1 patient had failed cannulation of the papilla. The rate of unsuccessful ERCP was (16\%). Regarding operative difficulties and complications, 8 patients (32\%) in our results had operative difficulties, 4 of them had acute cholecystitis and gall bladders were aspirated and omental adhesions were relieved by diathermy. 3 patients from this group (12\%) had the conversion to open cholecystectomy for CBD exploration. In the laparoscopic cholecystectomy, intra - operative cholangiogram group, 3 patients $(12 \%)$ were found to have choledocholithiasis, which was diagnosed during intraoperative cholangiogram. Open CBD exploration was done for the three patients and it was successful in the three patients to extract the stones. In the laparoscopic cholecystectomy, intra - operative cholangiogram group, 3 patients $(12 \%)$ were found to have choledocholithiasis, which was diagnosed during intraoperative cholangiogram. Open CBD exploration was done for the three patients and it was successful in the three patients to extract the stones. Regarding the operative difficulties and complications of patients of this group, in our results 6 patients (24\%) had operative difficulties. Regarding postoperative complications, our results showed only 1 complication (4\%), this patient had severe abdominal pain on 6 the post-operative day due to T- tube obstruction which was irrigated with saline, and he improved. No recorded cases of postoperative pancreatitis were found in the patients of this group in our results.
\end{abstract}

Conclusion: In our results, the preoperative ERCP with laparoscopic cholecystectomy group had the advantage of minimal invasive surgery.

Keywords: Pre-Operative, ERCP, Laparoscopic Cholecystectomy, Intraoperative Cholangiography, Acute Biliary Pancreatitis

\section{Introduction}

In all countries, acute pancreatitis is becoming more common. (1) Acute pancreatitis accounts for 3 percent of all 
cases of abdominal pain admitted to hospital. Stones of the common bile duct are the most important factor in acute pancreatitis. (2) Although majority of the patients with gallstone (biliary) pancreatitis recover without significant sequelae, $15-30 \%$ have severe episodes requiring multidisciplinary care to ensure the best outcome. De Beaux, Palmer, \& Carter, 1995; Trial, 1977) Complications of acute biliary pancreatitis, both local (necrosis, pseudo cyst formation, abscesses, hemorrhage) and systemic (pleural effusion, adult respiratory distress syndrome (ARDS), renal insufficiency, multiorgan failure) often require intensive care unit (ICU) management (Trial, 1977; McFadden, 1991).

Gender and stone size may be risk factors for gallstone pancreatitis. The risk of developing acute pancreatitis in patients with gallstones is greater in men; however, more women develop this disorder since gallstones occur with increased frequency in women. (Neoptolemos, London, James, Carr-Locke, Bailey, \& Fossard, 1988; Reila, Zinsmeister, \& Melton, 1991) Acute pancreatitis is a potentially fatal disease with an overall mortality rate of 2 7\% despite aggressive intervention. (De Beaux et al., 1995; Trial, 1977; Funnell, Bornman, Weakley, Terblanche, \& Marks, 1993) the outcome of acute pancreatitis is determined by two factors which reflect the severity of the illness: organ failure and pancreatic necrosis. About half of the deaths in patients with acute pancreatitis occur within the first one/two weeks and are mainly attributable to multiple organ dysfunction syndromes. When not treated, the risk of recurrence in gallstone pancreatitis ranges from 32 to 61\%. (Agarwal \& Pitchumoni, 1991; Pasricha, Kalloo, Clavien, Carroli, Phillips, Barritt Iii, \& Fan, 1993; De Beaux et al., 1995; Trial, 1977; Funnell, Bornman, Weakley, Terblanche, \& Marks, 1993; Gislason, Horn, Hoem, Andrén-Sandberg, Imsland, Søreide, \& Viste, 2004) the appropriate management of acute biliary pancreatitis has evolved considerably over the past decades. (Pasricha et al., 1993; De Beaux et al., 1995; Trial, 1977; Funnell et al., 1993; Gislason et al., 2004; Korman, Cosgrove, Furman, Nathan, \& Cohen, 1996; McFadden, 1991; Neoptolemos et al., 1988) Endolaparoscopic surgery plays a well-recognized role in the treatment of this pathology. Traditionally an episode of acute biliary pancreatitis is an indication for direct imaging of the biliary tree. The optimal approach may vary according to local expertise, and endoscopic retrograde cholangiopancreatography is the most common.

\section{Methods}

A Cohort study design used, the target population was patients with diagnosis of gall stone pancreatitis. The age ranged from 15 to 85 years (mean=50), 15 patients were males and 35 were females. They were studied before and after management.

During a period from January 2015 to December 2016, patients were selected randomly from the emergency department and surgical wards in King Fahad Hospital at Al Baha (Kingdom Saudi Arabia). All of them presented with acute gallstone pancreatitis. The total number of cases in this study is 50 patients. They were selected randomly and divided into 2 groups. The first group: 25 patients who had pre -operative ERCP followed by laparoscopic cholecystectomy ( 6 males and 19 females). The average age was 48 years old. The second group: 25 patients who had laparoscopic -cholecystectomy, intra operative cholangiogram and selective common bile duct exploration ( 9 males and 16 females) the average age was 47 years old as shown in Table 1.

Table 1. Demographics of patients

\begin{tabular}{|c|c|c|c|c|}
\hline & GROUP (1) & & GROUP (2) & \\
\hline Number of Patients & 25 & & 25 & \\
\hline Gender & 6 males & 19 females & 9 males & 16 females \\
\hline average age & $48 \mathrm{Y} / \mathrm{O}$ & & $47 \mathrm{Y} / \mathrm{O}$ & \\
\hline
\end{tabular}

\subsection{Pre-Operative Methods}

Every patient was subjected to history taking, physical examination, investigations including Complete Blood Count (CBC), chest X-ray, ECG, kidney function test, blood sugar level, liver function tests, serum amylase, coagulation profile, abdominal ultrasonography and Ranson`s criteria. Patient was admitted for few days to get resolution of symptoms and to be prepared for initial procedures like (ERCP or laparoscopic cholecystectomy and intraoperative cholangiogram).

\subsection{Intra-Operative Methods}

Subject was divided into two group as follow: 
Group (1): Pre-operative ERCP with endoscopic sphincterotomy if choledocholithiasis or dilated CBD were present. Then followed by laparoscopic cholecystectomy.

Group (2): Laparoscopic cholecystectomy, intraoperative cholangiogram or open cholecystectomy with selective common bile duct exploration.

\subsection{Post-operative Methods}

1)-Records of all operative intervention difficulties and complications were made.

2)-Close observation, recording of postoperative morbidities and mortalities.

3)-Total hospital length of stay

4)-Follow up all cases for three months.

5)-Statistics of all the results were made, computer assisted.

\section{Results}

According to groups of patients, Group (1) that was pre-operative ERCP with endoscopic sphincterotomy if choledocholithiasis or dilated CBD were present. Then followed by laparoscopic cholecystectomy, and Group (2) that was Laparoscopic cholecystectomy, intraoperative cholangiogram or open cholecystectomy with selective common bile duct exploration. There was variation in regards to clinical presentation in which $76 \%$ of patients of group 1 suffered from vomiting in comparison to group 2, however $52 \%$ of patients had jaundice in comparison to $68 \%$ of group 2 . In both groups there were positive signs of tenderness epigastric region and Right hypochondria with normal bowl sounds as shown in Table 2 .

Table 2. Clinical Presentation of Patients

\begin{tabular}{|c|c|c|c|c|c|c|c|c|}
\hline \multirow{2}{*}{ Patient } & \multirow{2}{*}{$\begin{array}{l}\text { Upper abdominal } \\
\text { pain referred to the } \\
\text { back }\end{array}$} & \multicolumn{2}{|l|}{ Vomiting } & \multicolumn{2}{|l|}{ Jaundice } & \multicolumn{2}{|c|}{ Tenderness $\quad$ epigastric } & \multirow{2}{*}{$\begin{array}{l}\text { Bowel } \\
\text { sound }\end{array}$} \\
\hline & & Positive & Negative & Positive & Negative & $\begin{array}{l}\text { region and } \\
\text { hypochondrium }\end{array}$ & Rt. & \\
\hline \multirow{3}{*}{$\begin{array}{l}\text { Group } \\
\text { (1) }\end{array}$} & \multirow{3}{*}{ positive } & 19 & 6 & 13 & 12 & \multirow{3}{*}{ positive } & & \multirow{3}{*}{ Normal } \\
\hline & & Patients & Patients & Patients & Patients & & & \\
\hline & & $76 \%$ & $24 \%$ & $52 \%$ & $48 \%$ & & & \\
\hline \multirow{3}{*}{$\begin{array}{l}\text { Group } \\
\text { (2) }\end{array}$} & \multirow{3}{*}{ Positive } & 22 & 3 & 17 & 8 & & & \multirow{3}{*}{ Normal } \\
\hline & & Patients & Patients & Patients & Patients & positive & & \\
\hline & & $88 \%$ & $12 \%$ & $68 \%$ & $32 \%$ & & & \\
\hline
\end{tabular}

\subsection{Abdominal Ultrasonography}

Table 3 shows a comparison between group 1 and 2 in regards to abdominal ultrasonography. In Group (1), there were 17 patients with normal liver, 5 with Dilated intrahepatic biliary radicles, 2 fatty livers, one cirrhotic, while in Group (2), there were 23 patients with normal liver, 2 with Dilated intrahepatic biliary radicles, in regards to pancreas status in there was 17 normal pancreases in both groups, 4 and 6 bulky, 2 and 1 prominent pancreatic duct respectively, with one case suffered from mild edema in group 1. Most of the cases in group one had multiple gallbladder stones. The average of CBD was higher in group two than one.

Table 3. A Comparison of Abdominal Ultrasonography Between Groups

\begin{tabular}{|c|c|c|}
\hline \multirow{2}{*}{ Abdominal Ultrasonography } & Group 1 & Group 2 \\
\hline & $\mathrm{N}=25$ & $\mathrm{~N}=25$ \\
\hline \multicolumn{3}{|l|}{ Liver } \\
\hline Normal & 17 & 23 \\
\hline Enlarged Fatty & 2 & 0 \\
\hline Cirrhotic & 1 & 0 \\
\hline Dilated intrahepatic biliary radicles & 5 & 2 \\
\hline
\end{tabular}


Pancreas

Normal

Bulky

Prominent Pancreatic duct

Mild edema

Obscured by intestinal gases

Dilated duct

Gall Bladder

Solitary stone

Contracted with multiple stones

Distended with multiple stone

Multiple stones

Multiple stones with G.B edema

CBD mm in Range
17

$\begin{array}{ll}17 & 17 \\ 4 & 6 \\ 2 & 1 \\ 1 & 1 \\ 1 & 0 \\ 0 & 0 \\ \end{array}$

17

\section{6}

1

0

0

1

$\begin{array}{ll}1 & 1 \\ 5 & 5 \\ 2 & 3 \\ 11 & 9 \\ 6 & 7 \\ 3.2-12,3 & 3.2-11\end{array}$

\subsection{Endoscopic Retrograde Cholangio-Pancreatography (ERCP)}

The mean time of doing ERCP after admission in this group was 3.4 days. Figure 1 shows that 8 patients were found to have filling defects (proved to be stones) (32\%) of group 1. 5 patients of them (20\%) stones were extracted during the ERCP, the remaining 3 patients $(12 \%)$ had failed ERCP to extract the stones. The only complication of ERCP done through the patients of this group (4\%) occurred with one of the patients with failure to extract the stones and some had retroperitoneal duodenal perforation. There was another 1 patient (4\%) who had failed cannulation of the papilla with failure of ERCP. There were 5 patients who had dilated CBD without stones $(20 \%)$. The remaining 11 patients had normal ERCP study. There was neither dilated CBD nor filling defects. Sphincterotomy was done in the first 13 patients $(52 \%)$ with extraction of stones in 5 patients $(20 \%)$, failure to extract stones in 3 patients $(12 \%)$, and dilated CBD without stones in 5 patients $(20 \%)$. There was no recorded incidence, of post ERCP pancreatitis among the patients of group 1 as shown in Figure 2.

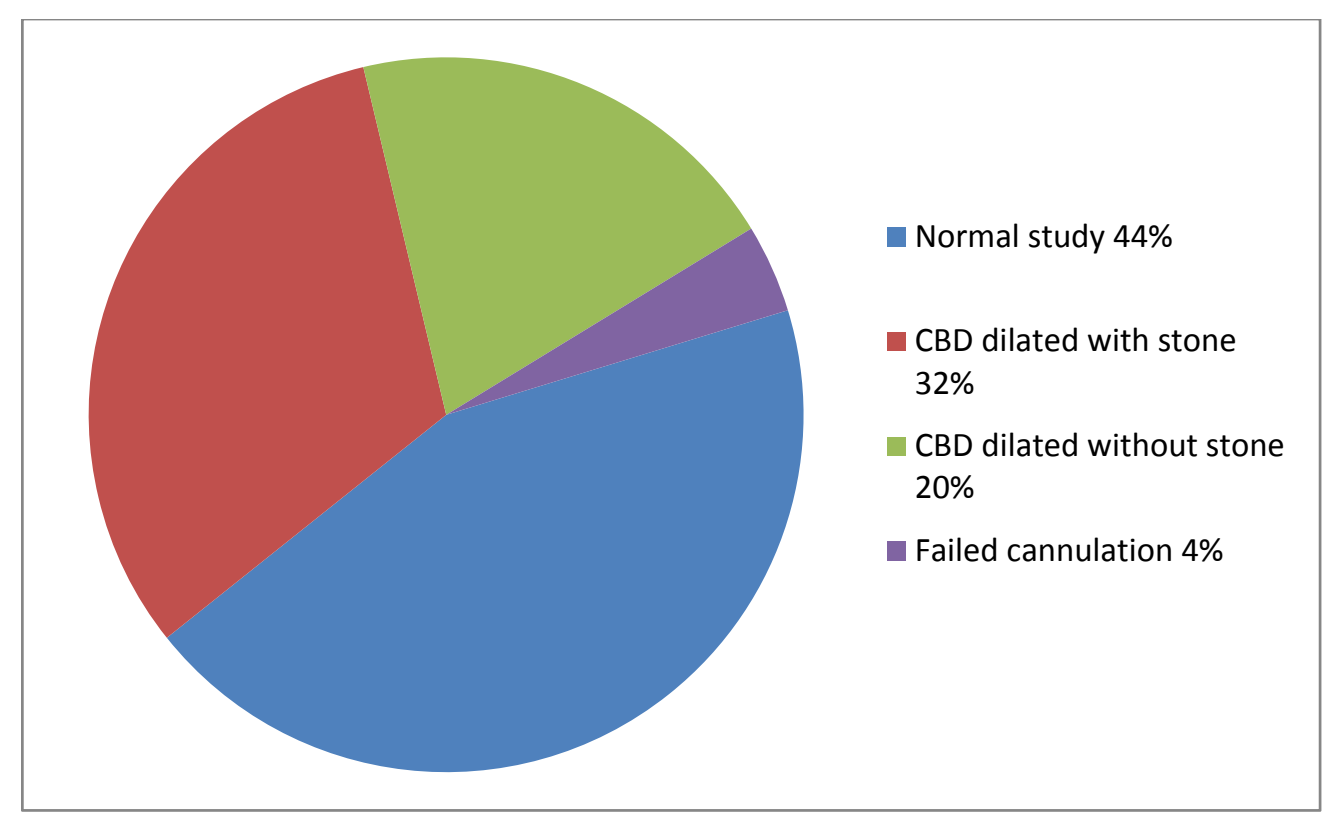

Figure 1. ERCP Results of Group 1 


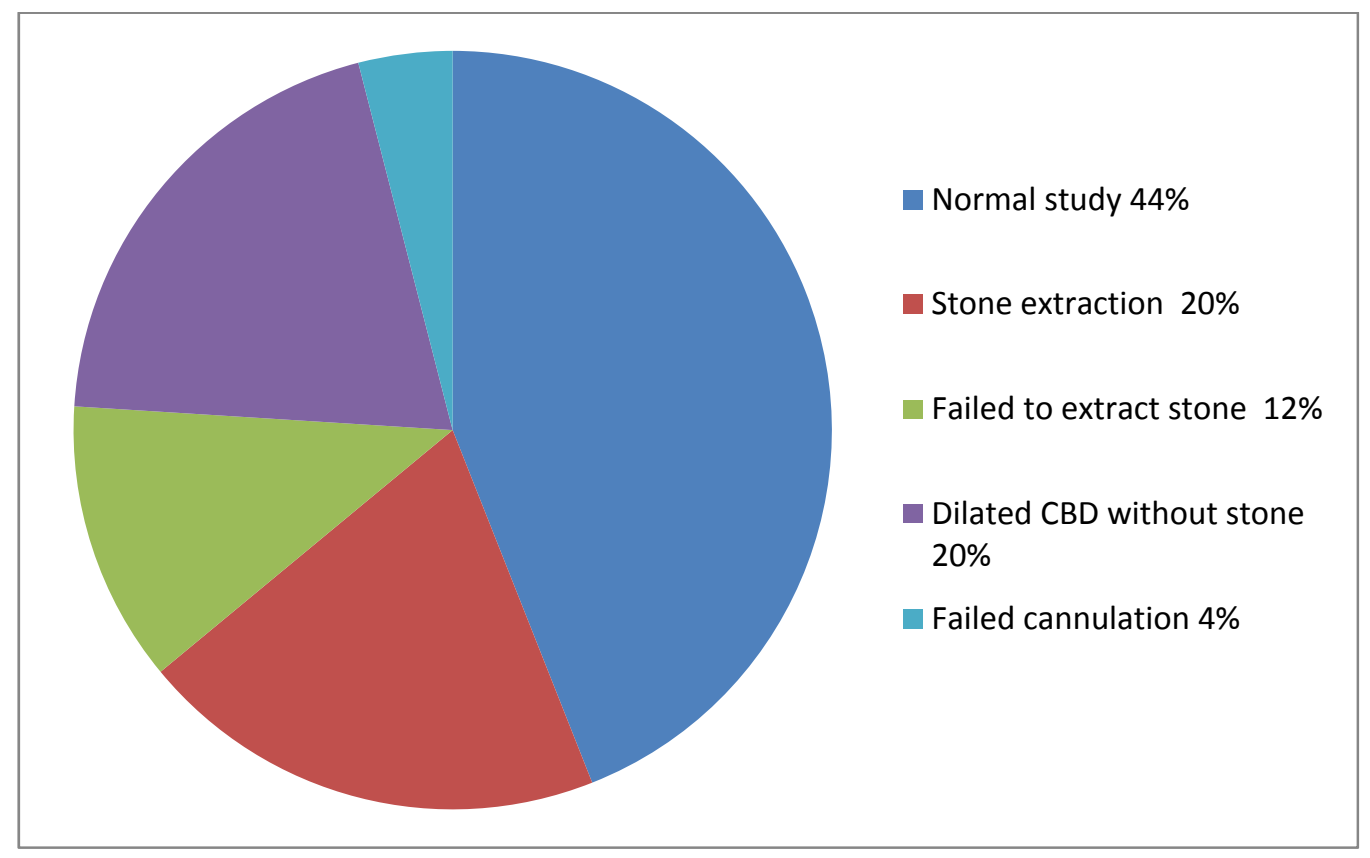

Figure 2. Rate of ERCP Failure in Group 1

\subsection{Laparoscopic Cholecystectomy for Patients of Group (1)}

The mean time of operation after ERCP was 3.8 days. Regarding operative difficulties and complications, figure 3. shows that 8 patients $(32 \%)$ had operative difficulties. Four of them had acute cholecystitis, the distended gallbladders were aspirated and omental adhesions were relieved by diathermy 3 patients $(12 \%)$ had conversion to open cholecystectomy for CBD exploration as shown in Figure 4. One of them had retroperitoneal duodenal perforation due to previous ERCP. Kocherization of the duodenum was done, collection of air and bile was found in retroperitoneal space, tube drain was fixed in Retroperitoneal space. Stones were removed from CBD either by Fogarty's catheter or stone forceps. 1 patient had multiple filling defects in the intraoperative cholangiogram. The gallbladder was removed laparoscopically. The patient had ERCP in the first postoperative day with removal of the stones. The other 17 patients $(68 \%)$ of this group the operation was completed laparoscopically without difficulties. 3 patients had post-operative complications (12\%), The patient who had retroperitoneal duodenal perforation had stormy postoperative period, She had acute renal failure, on the $4^{\text {th }}$ postoperative day urine was decreasing to $10 \mathrm{ml} /$ hour, serum urea $27 \mathrm{mmol} / 1$ and serum creatinine were $750 \mathrm{umol} / 1$. She was put on hemodialysis. After 20 days she was recovered from acute renal failure, urea and creatinine returned to normal levels. There were other patients who had post-operative wound infections treated with cleaning and dressing.

3.4 Laparoscopic Cholecystectomy, Intraoperative Cholangiogram and Selective CBD Exploration for Patients Group (2)

The mean time of operation after admission in this group was 6.2 days. Intraoperative cholangiogram revealed positive filling defects in the CBD in 3 patients (12\%) of this group as shown in Figure 5. The operation was converted to open procedure for CBD exploration. 2 other patients had conversion to open cholecystectomy without CBD exploration due to difficulty to perform the operation laparoscopically. The rate of conversion to open procedure in this group was 5 patients $(20 \%)$. 


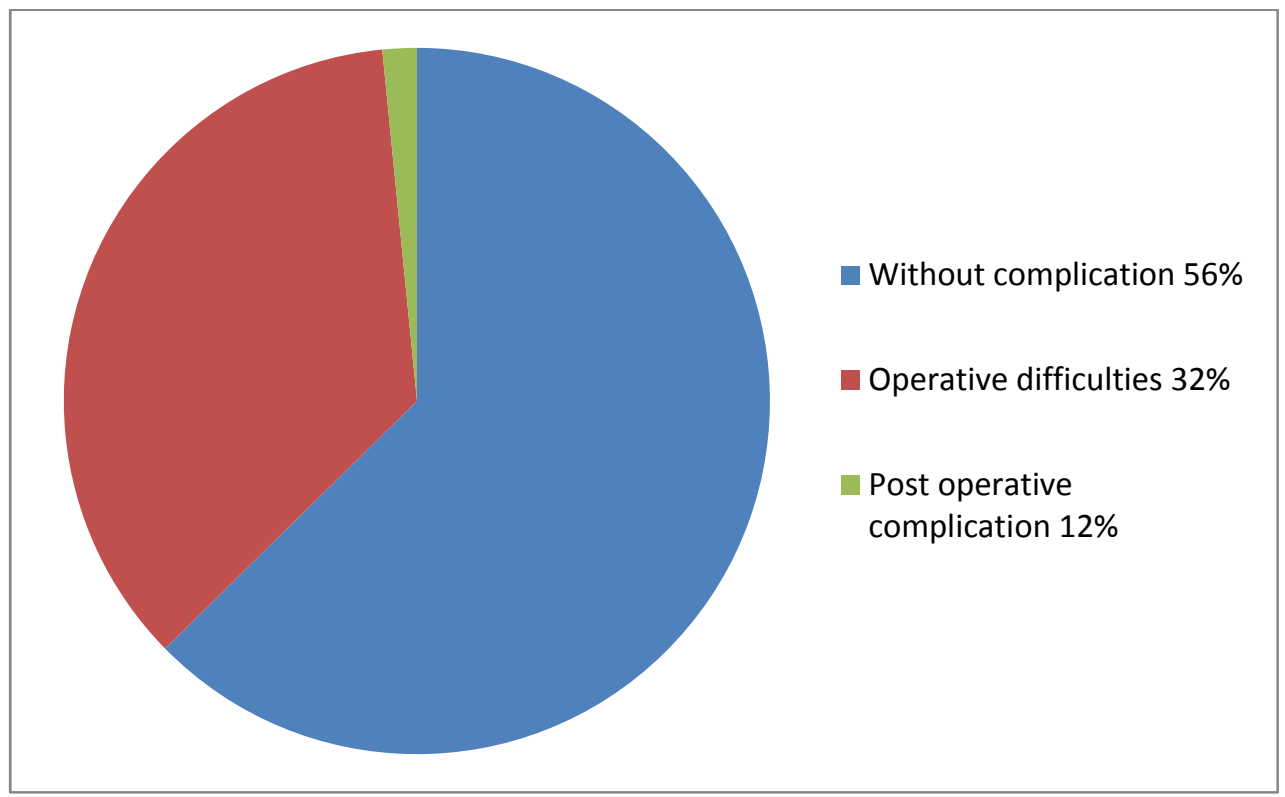

Figure 3. Operation of Group 1

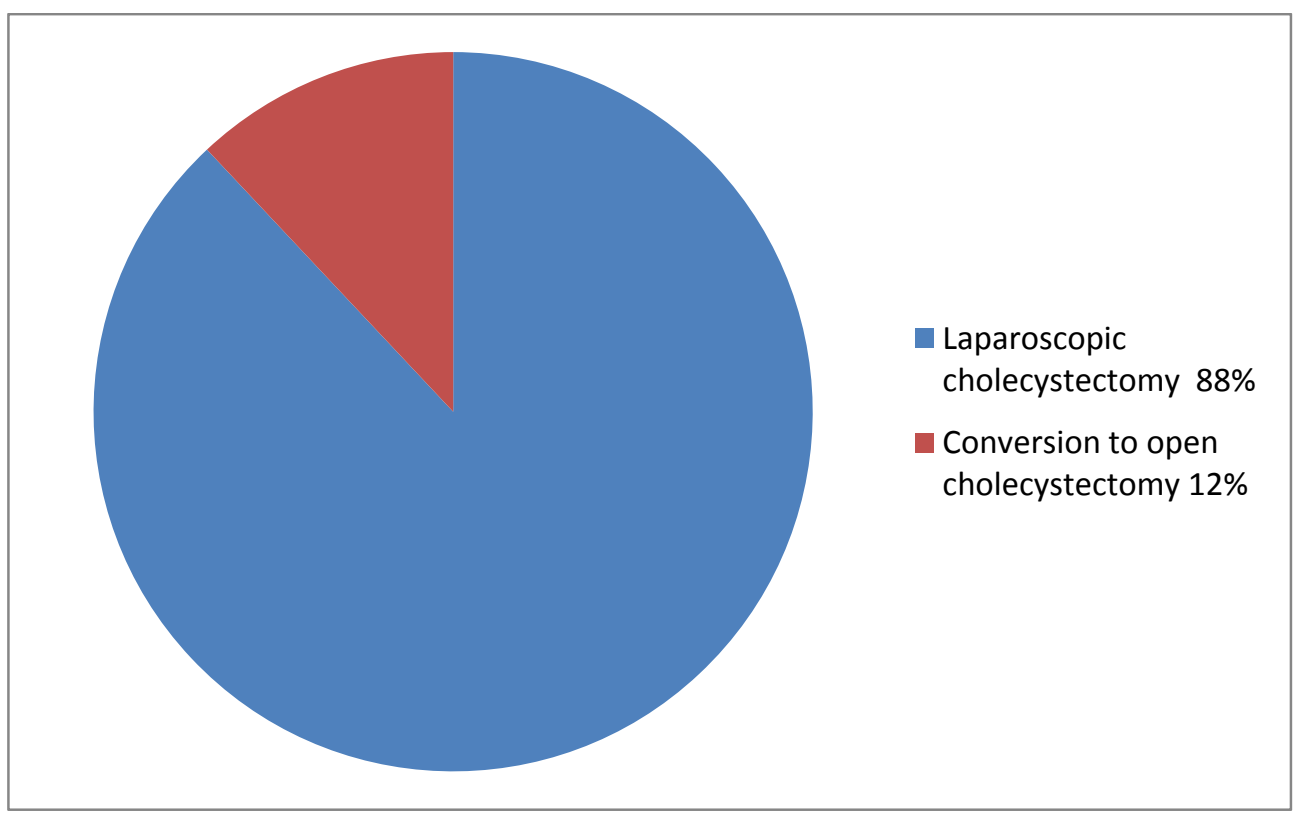

Figure 4. Conversion to open cholecystectomy in Group 1

\subsection{Operative Difficulties, Complications and Postoperative Complications of Patients of Group (2):}

The operative difficulties and complications of patients of this group, 6 patients $(24 \%)$ had operative difficulties as shown in Figure 6. One patient had difficulty of getting G.B out through the epigastric wound, which was extended. One patient had pancreatic cyst, which was aspirated laparoscopically, about 60-ml pus was aspirated.1 patient had hugely distended gallbladder, which was aspirated laparoscopically before starting the operation. 3 patients had stones inside the common bile duct, they were removed either by stone forceps or by Fogarty's catheter. Regarding the postoperative complications in this group Only 1 patient had postoperative complication on the 6 the post-operative day he had severe abdominal pain due to obstruction of T-Tube which was flushed and irrigated with saline, large amount of bile came out, patient gradually improved, T-tube was removed on $9^{\text {th }}$ Postoperative day after T-tube cholangiogram which revealed free flow of the dye to the duodenum. 
Length of hospital stay: Regarding the total length of hospital stay of patients of both groups. It was found that the mean total length of hospital stays of patients of group (1) was 12.32 days and group (2) was 10.8 days.

Follow up: Regarding the follow-up of patients of both groups for a period of 3 months after operation. All patients were seen regularly after 2 weeks, 1 month and 3 months. The follow-up period of patients of group (1) was good. No late postoperative complications were recorded in the patients of this group. Regarding the patients of group (2). 3 patients (12\%) from this group had recurrent attacks of acute pancreatitis during the first 2 months of follow-up period. All of them had ERCP which revealed normal Study in 2 patients, the third one had stone inside the common bile duct removed by ERCP.

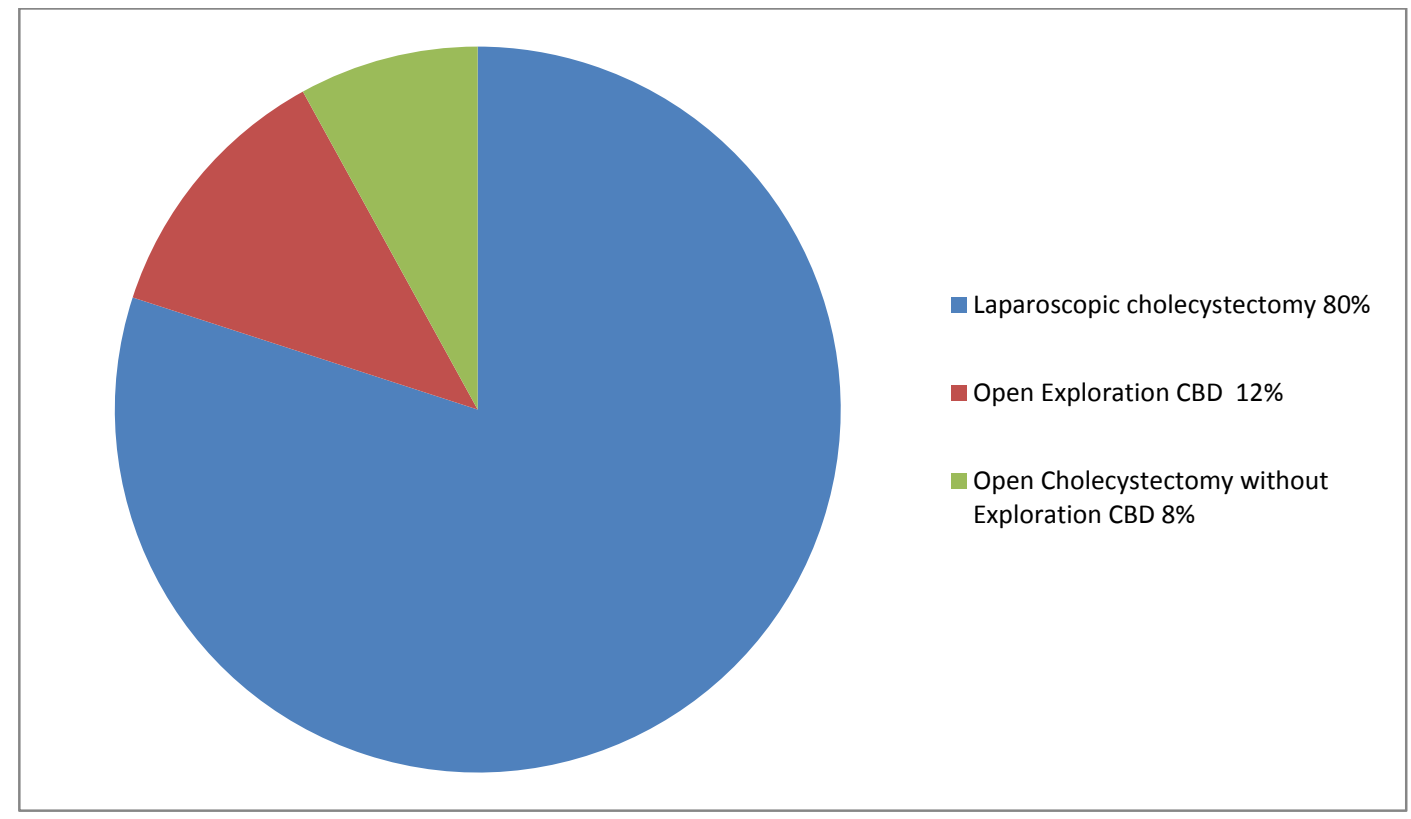

Figure 5. Operation of Group 2

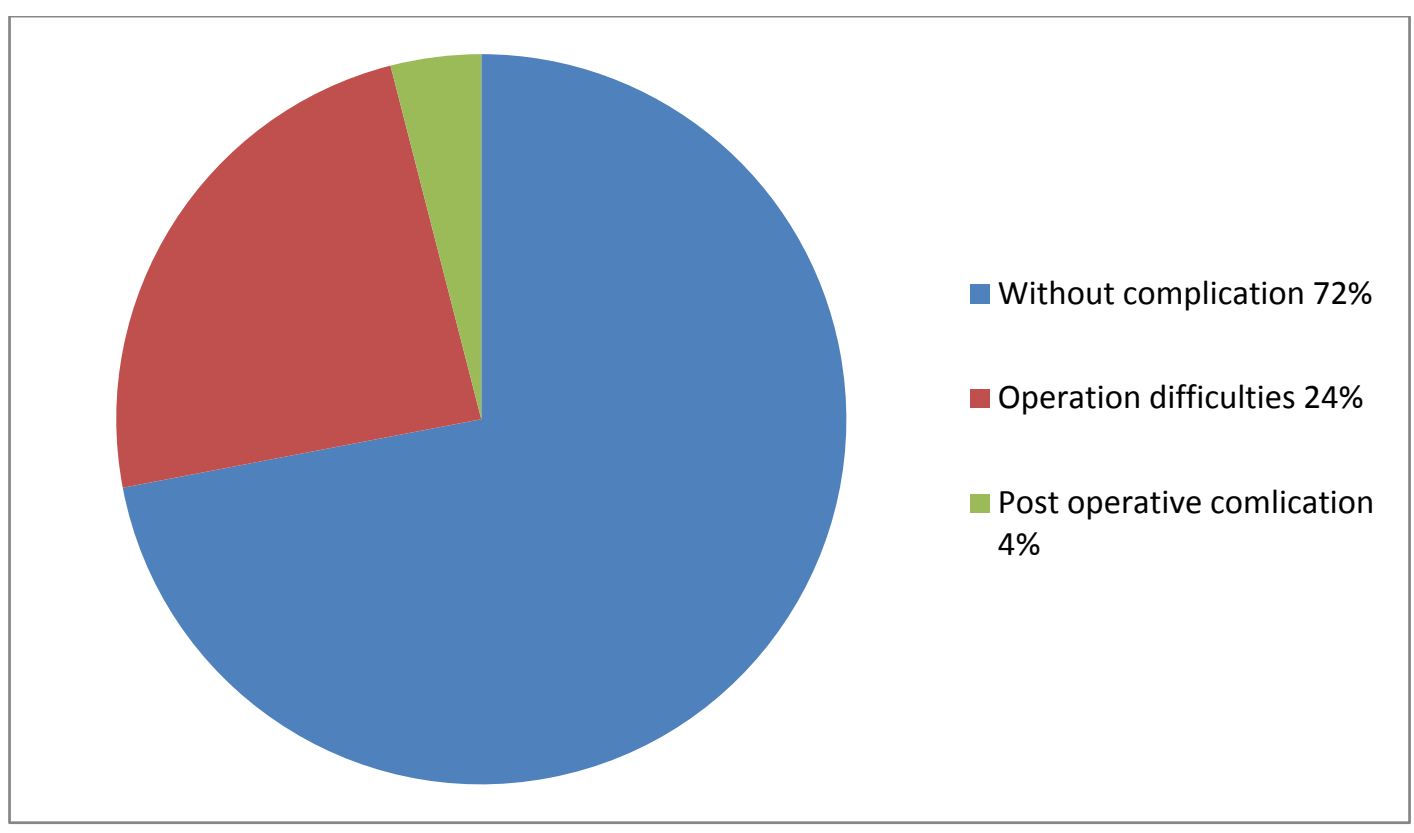

Figure 6. Operation Complications of Group 2 


\section{Discussion}

In our study, our patients involved in both groups, all had mild to moderate acute gallstone pancreatitis because all of them met 3 or less Ranson's criteria. All of these patients had significant hyper-amylasemia and associated liver function abnormalities, including individual enzyme elevations. Thirty-six patients of fifty $(72 \%)$ of the patients involved in both groups had obstructive jaundice on admission. In the preoperative ERCP and laparoscopic Cholecystectomy group, 8 patients (32\%) were found to have choledocholithiasis and underwent endoscopic sphincterotomy for stone extraction. Stones were extracted in 5 patients $20 \%$. The other 3 patients (12\%) ERCP failed to extract the stones. Another 1 patient had failed cannulation of the papilla. The rate of unsuccessful ERCP was (16\%).

In one study made by Sees and Martin, 1997 (Sees \& Martin, 1997), they reported that in the ERCP and laparoscopic cholecystectomy group (25\%) were found to have choledocholithiasis and underwent endoscopic sphincterotomy and stone extraction. The rate of Unsuccessful ERCP was $(10 \%)$ in their study.

In our study, dilated CBD without stones was found in five patients $(20 \%)$ on whom sphincterotomy was done. The remaining patients of these group 12 patients (48\%) the ERCP study was normal. Regarding operative difficulties and complications, 8 patients (32\%) in our results had operative difficulties, 4 of them had acute cholecystitis and gall bladders were aspirated and omental adhesions were relieved by diathermy. 3 patients from this group (12\%) had conversion to open cholecystectomy for CBD exploration.

In the other study, Sees and Martin 1997 (12), their results had a rate of conversion to open procedure in (10\%) of cases of this group. Regarding ERCP complications, in our results there was only one complication (4\%) in this group, this patient had retroperitoneal duodenal perforation. There were no recorded cases of post ERCP pancreatitis in our series.

In the other series, Sees and Martin 1997 they had (19\%) of cases developed symptomatic post ERCP pancreatitis. Regarding the post-operative complications in this group. 3 patients (12\%) had post-operative complications. The patient who had retroperitoneal duodenal perforation. She had post-operative acute renal failure which was treated by hemodialysis. The other two patients had post-operative wound. Infections treated by cleaning and dressing.

In the study done by korman et al. (1996), the recorded post-operative complication rate (10\%) of the patients included in the study. Regarding the length of hospital stay in this group, in our study it was 12.32 days. This length of hospital stay was due to the cases of unsuccessful ERCP and the case of complicated ERCP. Sees and Martin 1997 had mean length of hospital stay in this group 14.5 days; this was due to the cases that developed post FRCP pancreatitis which increased the length of their hospital stay. In the laparoscopic cholecystectomy, intra - operative cholangiogram group, 3 patients (12\%) were found to have choledocholithiasis, which was diagnosed during intraoperative cholangiogram. Open CBD exploration was done for the three patients and it was successful in the three patients to extract the stones. Sees and Martin 1997, their result showed (16\%) of their patients included in this group were found to have choledocholithiasis, all of them were treated with open CBD exploration except 1 patient who was treated with laparoscopic trans cystic duct exploration. In the laparoscopic cholecystectomy, intra - operative cholangiogram group, 3 patients (12\%) were found to have choledocholithiasis, which was diagnosed during intraoperative cholangiogram. Open CBD exploration was done for the three patients and it was successful in the three patients to extract the stones. In the series of Sees and Martin 1997, the rate of conversion to open procedure was also (20\%) of patients of this group including the cases opened for CBD exploration. Regarding the operative difficulties and complications of patients of this group, in our results 6 patients (24\%) had operative difficulties. 1 patient had difficulty of getting G.B out through the epigastric wound, which was extended. 1 patient had pancreatic cyst which was aspirated laparoscopically, $60-\mathrm{m} 1$ pus were aspirated. 1 patient had hugely distended gall bladder, which was aspirated laparoscopically before starting the operation. The other 3 patients had open CBD exploration for stone extraction. In the study of Korman et al 1996, he had a (10\%) rate of duct leak and three cases of retained CBD stones.

Regarding post-operative complications, our results showed only 1 complication (4\%), this patient had severe abdominal pain on 6 the post-operative day due to T- tube obstruction which was irrigated with saline, and he improved. No recorded cases of postoperative. Pancreatitis was found in the patients of this group in our results. Sees and Martin 1997, results showed that post-operative pancreatitis occurred in $(6 \%)$ of their patients included in this group. No other complications were identified in their patients.

Regarding the length of hospital stay in our results the mean length in this group was 10.8 days. Sees and Martin 
1997 (12), results were the same, their mean length was 10.1 days.

Regarding the follow up period for 3 months for all the patients. All patients of group (1) had good follow-up course without any complications. While three patients (12\%) of group (2) had recurrent attacks of acute Pancreatitis. One of them had CBD stone extracted during ERCP and the other two patients had normal ERCP study.

So, in our results, the preoperative ERCP with laparoscopic cholecystectomy group had the advantage of minimal invasive surgery. Although the intraoperative difficulties and complications were more than the laparoscopic cholecystectomy and intra-operative cholangiogram group, the conversion rate to open procedure was less than the second group. The early post-operative complications were the same in both groups.

\section{Conclusion}

Gallstone pancreatitis is caused by transient obstruction of the ampulla of Vater by a migrating gallstone. Intraglandular activation of pancreatic enzymes occurs (by an unclear mechanism), and their entry into the circulation causes most of the local and systemic events of pancreatitis.

The diagnosis is based on history and physical examination, an elevation of serum amylase above $1000 \mathrm{IU} / \mathrm{L}$, and ultrasound and CT scans. Endoscopic retrograde cholangiopancreatography can be used to confirm the presence of common bile duct stones. Because of the absence of an agent that can abort progression of the disease, therapy should consist of adequate resuscitation, nutritional support, and careful monitoring to detect early complications. Pre-operative ERCP/endoscopic sphincterotomy in conjunction with laparoscopic cholecystectomy, and laparoscopic cholecystectomy with intra-operative cholangiogram and common bile duct exploration are effective ways to manage patients with gallstone pancreatitis. Although all of our patients had mild to moderate gallstone pancreatitis with a rapid clinical and chemical recovery, which would predict a low likelihood of choledocholithiasis, we found a high incidence of choledocholithiasis in our group of patients $(20 \%)$.

The present study, suggests that ERCP-ES can be safely performed in patients with acute pancreatitis, but only by a person with skill and considerable experience.

This treatment is especially performed in those patients who have biochemical evidence of biliary obstruction (i.e. increased bilirubin levels and increased alkaline phosphatase level, etc.) or who have direct and indirect ultrasonographic evidence of choledocholithiasis. If Choledocholithiasis is proven during the performance of ERCP, and then ES should be performed in the same sitting. If this strategy is followed, then we would recommend performing a laparoscopic cholecystectomy during the same admission, as soon as the symptoms of acute pancreatitis have subsided.

This treatment decreases the chances of complications, improves pain and decreases the hospital stay. On the other hand, if early ERCP has not been done, we would recommend performing a laparoscopic cholecystectomy a day or two prior to leave from the hospital. This strategy minimizes the need for common bile duct exploration and still achieves the goal of a limited hospital stay (the patient can go home a day after the laparoscopic procedure) and the prevention of future attacks of pancreatitis.

Under these circumstances, however, it is imperative that an operative cholangiogram of good quality be obtained during the laparoscopic cholecystectomy; since the risk of associated choledocholithiasis is still relatively high (about 20\%) and residual choledocholithiasis will result in recurrence of acute pancreatitis within 6 months in nearly all patients. If choledocholithiasis is found during the performance of laparoscopic cholecystectomy, it should be treated laparoscopically, if at all possible. If this is not feasible, we believe that, for the average surgeon, it may be best to decide either to convert the laparoscopic procedure to an open procedure or to treat the choledocholithiasis by ERCP-ES the next day.

\section{Acknowledgements}

I wish to express my sincere gratitude and deep appreciation to Professor Dr. Catalan Vasilescu, professor of General surgery, Carol Davila University for giving me the opportunity to perform this study, his guidance, invaluable help, kind directions, supervision, generous support and continuous encouragement.

\section{Competing Interests Statement}

The authors declare that there is no conflict of interests regarding the publication of this paper.

\section{References}

Agarwal, N., \& Pitchumoni, C. S. (1991). Assessment of severity in acute pancreatitis. American journal of 
gastroenterology, 86(10).

De Beaux, A. C., Palmer, K. R., \& Carter, D. C. (1995). Factors influencing morbidity and mortality in acute pancreatitis; an analysis of 279 cases. Gut, 37(1), 121-126. https://doi.org/10.1136/gut.37.1.121

Funnell, I. C., Bornman, P. C., Weakley, S. P., Terblanche, J., \& Marks, I. N. (1993). Obesity: an important prognostic factor in acute pancreatitis. British journal of surgery, 80(4), 484-486. https://doi.org/10.1002/bjs.1800800426

Gislason, H., Horn, A., Hoem, D., Andrén-Sandberg, Å., Imsland, A. K., Søreide, O., \& Viste, A. (2004). Acute pancreatitis in Bergen, Norway A study on incidence, etiology and severity. Scandinavian journal of surgery, 93(1), 29-33. https://doi.org/10.1177/145749690409300106

Hazem, Z. M. (2009). Acute biliary pancreatitis: diagnosis and treatment. Saudi journal of gastroenterology, 15(3), 147. https://doi.org/10.4103/1319-3767.54740

Korman, J., Cosgrove, J., Furman, M., Nathan, I., \& Cohen, J. (1996). The role of endoscopic retrograde cholangiopancreatography and cholangiography in the laparoscopic era. Annals of surgery, 223(2), 212. https://doi.org/10.1097/00000658-199602000-00015

McFadden, D. W. (1991). Organ failure and multiple organ system failure in pancreatitis. Pancreas, 6, S37-S43. https://doi.org/10.1097/00006676-199101001-00007

Neoptolemos, J. P., London, N. J., James, D., Carr-Locke, D. L., Bailey, I. A., \& Fossard, D. P. (1988). Controlled trial of urgent endoscopic retrograde cholangiopancreatography and endoscopic sphincterotomy versus conservative treatment for acute pancreatitis due to gallstones. The Lancet, 332(8618), 979-983. https://doi.org/10.1016/S0140-6736(88)90740-4

Pasricha, P. J., Kalloo, A. N., Clavien, P. A., Carroli, B. J., Phillips, E. H., Barritt Iii, A. S., \& Fan, S. T. (1993). The early treatment of acute biliary pancreatitis. New England Journal of Medicine.

Reila, A., Zinsmeister, A. R., \& Melton, L. J. (1991). Etiology, incidence and survival of acute pancreatitis (AP) in Olmsted, Minnesota. Gastroenterology, 100, A296.

Sees, D. W., \& Martin, R. R. (1997). Comparison of preoperative endoscopic retrograde cholangiopancreatography and laparoscopic cholecystectomy with operative management of gallstone pancreatitis. The American journal of surgery, 174(6), 719-722. https://doi.org/10.1016/S00029610(97)00216-X

Trial, M. M. (1977). Death from acute pancreatitis. Lancet, 2, 632-635.

\section{Copyrights}

Copyright for this article is retained by the author(s), with first publication rights granted to the journal.

This is an open-access article distributed under the terms and conditions of the Creative Commons Attribution license (http://creativecommons.org/licenses/by/4.0/). 